\title{
Mannose: a marker for adulteration with syrup or resin treatment of blossom honey
}

J. Missler ${ }^{a^{*}}$, T. Wiezorek and G. Beckh ${ }^{a}$

${ }^{a}$ Quality Services International GmbH, Flughafendamm 9a, 28199 Bremen: Corresponding Author: Jane.Missler@qsi-q3.de

Adulteration has become an issue on the honey market in the past years and may occur unintentionally through feeding during nectar flow or by adding sugars/syrup to the honey. To ensure the quality and authenticity of honey it needs to be tested. For analysis of honey the availability of specific markers for processing, quality or especially for adulteration are important. The F/G ratio (fructose/ glucose ratio) of a unifloral honey can be specific for its botanical origin. A F/G ratio $<1.0$ is rare and normally typical for rape (Brassica napus, also called canola) honey, additionally it could be observed that rice syrups have similar F/G values as rape honey. One particular marker for sugar adulteration in blossom honey is mannose. All measured natural blossom honeys showed no indication of mannose. We found out that mannose finds its way into blossom honey either through adulteration with high end syrups or through treatment with ion exchange resins as part of purifying processes.

\section{Introduction}

uthenticity of honey has become an important issue for the honey industry in the past years. The European Union (EU) currently endeavours to get an overview of the so-called economically motivated adulteration (EMA) of honey. In 2015 European honey samples were collected and tested by the European Commission's Joint Research Centre - Institute for Reference Materials and Measurements (JRC-IRMM) and 19\% of them showed incongruities, e.g. in their chemical-physical parameters, foreign sugars or their botanical and geographical origin ${ }^{1}$. Adulterations can appear unintentionally through feeding during nectar flow or by adding sugars/syrup to the honey. To ensure the quality and authenticity of honey it needs to be tested. Specific markers for processing, quality or especially for adulteration are important for the conventionally honey analyses and regulated by the European Union (EU Directive 110/2001)2. 5-(Hydroxymethyl)furfural (HMF) for example is a quality and processing marker, which indicates storage conditions, the age of honey or heating as part of processing and occurs through acidic hydrolysis of hexoses ${ }^{3}$. Therefore several analyses need to be conducted, each are often only specific for one adulterant.

The new approach is the "Honey-Profiling ${ }^{\text {TM" }}$ using nuclear magnetic resonance spectroscopy $(\mathrm{NMR})^{4}$. It is based on the Bruker FoodScreener (Avance III HD $400 \mathrm{MHz}$ ) and was developed by Bruker Biospin $\mathrm{GmbH}$ (Rheinstetten, Germany) in cooperation with ALNuMed GmbH (Bayreuth, Germany) and Quality Services International GmbH (QSI Bremen, Germany) $)^{5}$. The Version 1.0.0 of the profiling comprised non-targeted verification analysis and the quantification of 36 parameters, e.g. sugars, amino and organic acids in comparison with the NMR reference database, representing more than 3600 authentic honey samples from more than 50 different countries and 20 botanical varieties. The non-targeted verification analysis (chemometrics) consists of the indications for addition of syrup/sugar through indices (ratios) of specific substances or spectrum sections and the univariate and multivariate verification. With the univariate verification, chemical shift areas are compared with the database and peaks which are too high, too small, missing or completely new in the spectrum are mentioned. The multivariate verification is based on the comparison of the NMR parameters of the specific sample and those of the database for the particular model in a multi-dimensional space using Mahalanobis distance ${ }^{6}$. The samples can be compared with different models for geographical origins or botanical varieties. The authenticity of all samples was verified through more than 44000 conventional analyses, e.g. microscopical pollen analysis, foreign enzymes like $\alpha$-amylase, ${ }^{13} \mathrm{C}$-IRMS for $\mathrm{C}_{4}$-plant syrup adulteration or the analysis of beetroot and rice syrup markers.

Another new marker for sugar/syrup adulteration is the monosaccharide mannose (C2-epimer of glucose), which was identified and used as marker by QSI. It is found in nature as polysaccharide in glucomannans and glucoproteins of fruits like cranberries and black current or herbs or Aloe vera ${ }^{7,8}$. Mannose does not naturally appear in blossom honey but can be found in honeydew honeys depending on their geographical and botanical origin ${ }^{9}$. If a blossom honey contains mannose it is considered adulterated. The question is: How does mannose find its way into the blossom honey? To gain more information on the occurrence and formation of mannose in honey we conducted experiments with honey and syrups based on common honey processing procedures.

\section{Experimental \\ Sample preparation}

The sample preparation method for honey was adapted from Bruker Biospin GmbH (Rheinstetten, Germany). The homogenized honey and syrup samples $(5 \mathrm{~g})$ were diluted in $17.5 \mathrm{ml}$ NMR-buffer $\left(\mathrm{KH}_{2} \mathrm{PO}_{4}\right.$, Merck; $\mathrm{NaN}_{3}$, Fluka) and adjusted to a $\mathrm{pH}$ value of 3.1 with $\mathrm{HCl}$ (Chemsolute) for routine measurements. The honey and syrup samples for the mannose transformation were mixed with $230 \mu \mathrm{l}$ $0.8 \mathrm{M} \mathrm{NaOH}$ (Merck) to acquire an alkaline $\mathrm{pH}$ value $(\mathrm{pH} 9-10)$ and were incubated for 48 hours at $37^{\circ} \mathrm{C} .900 \mu$ l of the homogenized honey and syrup solutions were taken and $100 \mu$ standard solutions $\left(0.1 \%\right.$ trimethylsilylpropanoic acid, TSP, Alfa Aesar; in $\mathrm{D}_{2} \mathrm{O}$, 
Sigma-Aldrich) were added. The final sample solution was centrifuged and transferred into a $5 \mathrm{~mm}$ NMR-tube (Deutero) for direct measurement.

\section{${ }^{1}$ H-NMR measurement}

All measurements were performed on a Bruker Avance III HD $400 \mathrm{MHz}$ equipped with a $5 \mathrm{~mm}$ PA BBI $400 \mathrm{SI} \mathrm{H}$-BB-D-05 Z probe. ${ }^{1} \mathrm{H}$-NMR-spectra were acquired at $300.2 \mathrm{~K}$ using the pulse program noesygppr1d (1D spectra with water presaturation at $4.8 \mathrm{ppm}$ ). All spectra were automatically phased, baseline-corrected, and calibrated using TSP as reference at $0.0 \mathrm{ppm}$. The compounds were quantified by using the Honey-Profiling ${ }^{\mathrm{TM}}$ routine (Release 1.0, Bruker Biospin $\mathrm{GmbH}$, Rheinstetten, Germany) by automatic integration of the peak area calculated with an external standard (Spraul et al. 2009) $^{10}$.

\section{Results and discussion}

To this day more than 5300 honeys and 50 syrups from different geographical origins and varieties were measured with the HoneyProfiling $^{\mathrm{TM}}$ and were evaluated according to EU Directive 110/2001 into typical (natural) and untypical (not natural) honey. We observed that a lot of these untypical honeys are not originated in Europe. Table 1 shows the fructose $(F)$, glucose $(G)$ and mannose contents and the $F / G$ ratios of Chinese honeys and rice syrups as example.

Fructose and glucose are the main sugars besides some smaller amounts of di-, tri- and oligosaccharides in honey. The ratio F/G is an important honey parameter and specific for botanical varieties. Except of rape (Brassica napus) honey all other unifloral honeys show an F/G >1.0. An acacia (Robinia pseudoacacia) honey for example has one of the highest F/G-ratios $(1.5-1.7)^{11}$.

The honeys and syrups in Table 1 show different sugar concentrations depending on their origin and processing. It was observed that the syrups have an F/G lower than 1.0. Additionally, the natural fennel (Foeniculum spec.), acacia and vitex (Vitex angustifolia) honeys contain no mannose in comparison to the resin treated, adulterated (with high end syrup) honeys and processed syrups.

Mannose can be observed in honeys and in high end syrups (e.g. purified fructose/glucose syrups) ${ }^{12}$, especially in products coming from China. It finds its way into blossom honey through either adulteration with high end syrups containing mannose or through purifying processes with ion exchange resins (see Figure 1).

Syrups and honeys are cleaned/purified with special ion exchange resins ${ }^{13}$ to eliminate unwanted substances, e.g. HMF, pesticides or antibiotics. There are different types of ion exchange resins which are cross-linked solid polymer chains carrying ionic groups, like $\mathrm{SO}_{3}^{-}, \mathrm{COO}^{-}, \mathrm{NH}_{3}^{+}, \mathrm{NH}_{2}^{+}$. Depending on the types of ions (positive or negative) or if there is no charge involved a cation, anion or neutral exchanger with a particular $\mathrm{pH}$ value (basic, acidic, neutral) is needed $^{14}$. In order to gain more information about the honey processing with resins, we incubated different honeys and syrups with $\mathrm{NaOH}$ to simulate an anion exchange process (see Table2).

The results show that both the fructose- and glucose concentrations decrease and mannose and other products, like carbohydrates and organic acids appear. The F/G consequently changes because of the different consumption of fructose and glucose. Monosaccharides, like glucose, undergo under alkaline or acidic conditions or through enzymatic isomerase reaction an aldose ketose isomerisation (keto-enol tautomerism) ${ }^{15,16}$ (see Figure 2).

The aldose (glucose) converts into a 1,2-enediol intermediate via deprotonation and with a following protonation into a ketose (fructose). Fructose transforms via trans and cis 1,2-enediol intermediate into glucose and mannose.

Because of its distinct appearance in syrups and honeys, mannose is an important parameter indicating an adulteration with exogenous sugars or processing with ion exchange resins. In 2015, about $8 \%$ of all measured blossom honeys contain mannose. Nevertheless the absence of mannose does not consequently mean that the honey is a natural product without adulteration or processing. Beetroot syrup for instance does not show any mannose neither does feeding with sugar or special bee feed. Therefore the mannose content should never be evaluated solely but rather in conjunction with the Honey-Profiling ${ }^{\mathrm{TM}}$ in order to distinguish between adulterations, processing or blends with mannose containing honey dew honeys.

\section{Conclusion}

Mannose finds its way into blossom honeys either through adulteration with high end syrups or through treatment with ion exchange

Table 1. Sugar quantities of natural ripe blossom honey, honeys treated with resin, ripe honeys adulterated with syrup and high end rice syrup.

\begin{tabular}{|c|c|c|c|c|}
\hline Samples & Fructose (F) [g/100g] & Glucose (G) [g/100g] & F/G & Mannose $[\mathrm{g} / 100 \mathrm{~g}]$ \\
\hline Natural Chinese Fennel Honey & 39.5 & 26.7 & 1.47 & 0 \\
\hline Natural Chinese Acacia Honey & 40.2 & 27.4 & 1.47 & 0 \\
\hline Natural Chinese Vitex Honey & 34.0 & 27.6 & 1.27 & 0 \\
\hline Polyfloral Honey with resin & 35.5 & 31.0 & 1.15 & 0.02 \\
\hline Acacia Honey with resin & 41.3 & 27.9 & 1.48 & 0.03 \\
\hline Vitex Honey with resin & 36.5 & 29.7 & 1.23 & 0.06 \\
\hline Chinese Fennel Honey with syrup & 33.8 & 33.2 & 1.02 & 0.07 \\
\hline Chinese Acacia Honey with syrup & 45.2 & 28.9 & 1.56 & 0.07 \\
\hline Chinese Vitex Honey with syrup & 44.1 & 31.6 & 1.39 & 0.07 \\
\hline Rice syrup 1 (processed) & 34.0 & 37.8 & 0.90 & 0.06 \\
\hline Rice syrup 2 (processed) & 29.7 & 32.9 & 0.90 & 0.09 \\
\hline Rice syrup 3 (processed) & 34.8 & 35.4 & 0.98 & 0.09 \\
\hline
\end{tabular}




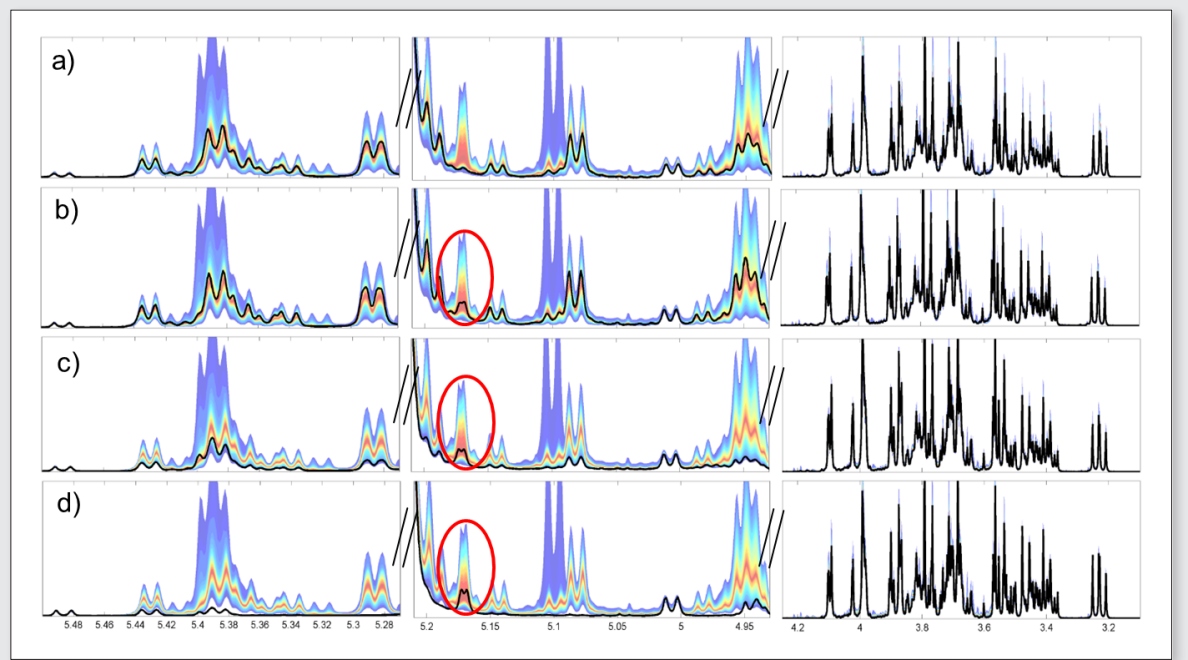

Figure 1. Overview of the sugar sections of Honey-Profiling ${ }^{\text {TM }}$ quantilplot areas ( $\left.3-5 \mathrm{ppm}\right)$ : a) a ripe Chinese Vitex honey, b) a ripe Chinese Vitex honey with mannose (5.17 ppm) treated with resin, c) a ripe Chinese Vitex honey adulterated with rice syrup with mannose, d) rice syrup containing mannose.

resins as part of a purifying process. It proved to be a well suited indicator for some additions of exogenous sugars or processing with ion exchange resins. However it is just a single marker and the evaluation should be supported in context with the HoneyProfiling $^{\mathrm{TM}}$ in order to get comprehensive information of adulteration practices which are not indicated by mannose. Only one fast

Table 2. Sugar quantities of natural honey and syrups after $\mathrm{Oh}$ and $48 \mathrm{~h}$ incubation with $\mathrm{NaOH}$ at $37^{\circ} \mathrm{C}$.

\begin{tabular}{|c|c|c|c|c|}
\hline Samples & Fructose (F) [g/100g] & Glucose (G) [g/100g] & $F / G$ & Mannose $[\mathrm{g} / 100 \mathrm{~g}]$ \\
\hline Beetroot Syrup, China, Oh & 34.6 & 33.2 & 1.04 & 0 \\
\hline Beetroot Syrup, China, 48h & 14.9 & 15.2 & 0.98 & 0.39 \\
\hline Rice Syrup 1, China, Oh & 0.2 & 15.1 & 0.02 & 0 \\
\hline Rice Syrup 1, China, 48h & 0.2 & 7.6 & 0.03 & 0.05 \\
\hline Rice Syrup 2, China, Oh & 0.2 & 21.4 & 0.01 & 0 \\
\hline Rice Syrup 2, China, 48h & 0.3 & 8.8 & 0.03 & 0.03 \\
\hline Organic Brown Rice Syrup, China, Oh & 0.2 & 14.4 & 0.01 & 0 \\
\hline Organic Brown Rice Syrup, China, 48h & 0.3 & 6.7 & 0.04 & 0.05 \\
\hline Vitex Honey 1 , China, Oh & 40.5 & 34.7 & 1.17 & 0 \\
\hline Vitex Honey 1 , China, $48 \mathrm{~h}$ & 20.1 & 17.8 & 1.13 & 0.06 \\
\hline Fennel Honey, China, Oh & 39.3 & 28.6 & 1.38 & 0 \\
\hline Fennel Honey, China, 48h & 20.0 & 14.6 & 1.37 & 0.03 \\
\hline Vitex Honey 2, China, Oh & 35.7 & 31.3 & 1.14 & 0 \\
\hline Vitex Honey 2, China, 48h & 18.0 & 16.0 & 1.12 & 0.02 \\
\hline Polyfloral Honey, China, Oh & 37.1 & 32.4 & 1.15 & 0 \\
\hline Polyfloral Honey, China, 48h & 17.0 & 15.1 & 1.13 & 0.11 \\
\hline Organic Acacia, Germany, Oh & 43.1 & 27.1 & 1.59 & 0 \\
\hline Organic Acacia, Germany, 48h & 21.2 & 14.3 & 1.49 & 0.04 \\
\hline Forest Honey, Italia Oh & 36.2 & 34.5 & 1.05 & 0 \\
\hline Forest Honey, Italia 48h & 13.1 & 13.7 & 0.95 & 0.04 \\
\hline Yucatan Honey, Mexico 48h & 37.3 & 32.0 & 1.16 & 0 \\
\hline Yucatan Honey, Mexico 48h & 17.1 & 14.5 & 1.18 & 0.03 \\
\hline Acacia, Romania 48h & 41.4 & 25.0 & 1.66 & 0 \\
\hline Acacia, Romania 48h & 20.8 & 13.9 & 1.50 & 0.06 \\
\hline
\end{tabular}




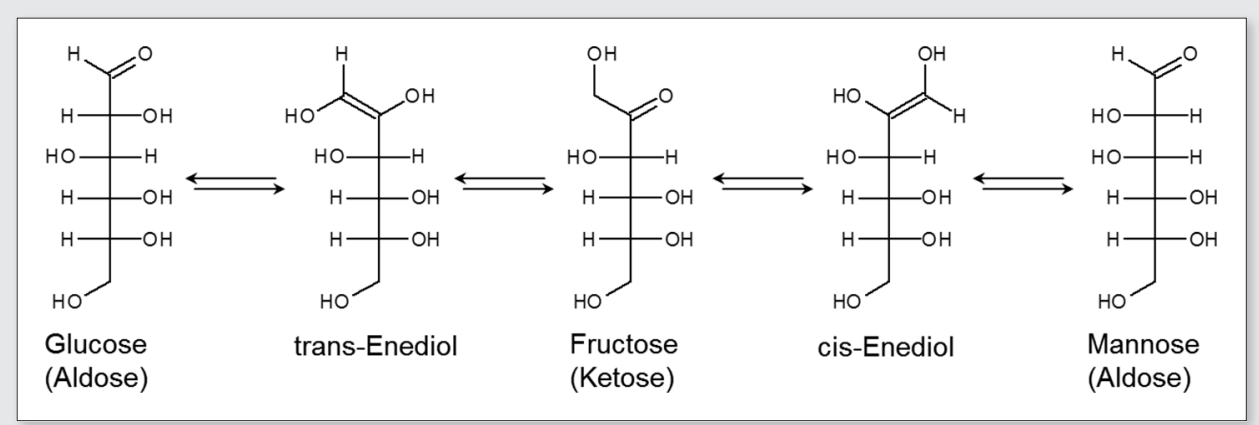

Figure 2. Lobry-de-Bruyn-Alberda-van-Ekenstein transformation: base catalysed aldose ketose isomerisation of D-glucose, D-fructose and D-mannose via 1,2-enediol intermediate.

measurement is necessary to get comprehensive data which can also be evaluated in new adulteration context retrospectively. The evaluation of mannose as part of it is important and useful for honey of certain geographical origins, where ion exchange processing for honey and mannose containing syrups for adulteration are commonly used. Moreover it is important to investigate the purifying processes with different ion exchange resins in order to gain more knowledge about this particular processing step. Further honey adulteration experiments with high end syrups of different origins should be conducted, to observe the changes in the sugar composition and to find new specific marker substances.

\section{References}

1. European Commission's Joint Research Centre (JRC-IRMM), "Commission recommendation of 12.3.2015 on a coordinated control plan with a view to establishing the prevalence of fraudulent practices in the marketing of certain foods", (2015). doi: http://ec.europa.eu/food/ safety/docs/official-controls_food-fraud_fish_recom-2015-1558_actannexes_en.pdf

2. The Council of the European Union, "Council Directive 2001/110/EC of 20 December 2001 relating to honey", Official Journal of the European Communities L10, 47-52 (2001). doi: http://eurlex.europa.eu/ LexUriServ/LexUriServ. do?uri=OJ:L:2002:010:0047:0052:EN:PDF

3. M. Zappalà, B. Fallico, E. Arena and A. Verzera, "Methods for the determination of HMF in honey: a comparison", Food Control 16, 273277 (2005). doi: https://www.researchgate.net/publication/222686159 Methods_for_the determination_of_HMF_in_honey_A_comparison

4. A. Dübecke, "NMR-Profiling of Honey - The New Approach in Honey Authenticity Testing", eFoodLab International 3, 14-16 (2015).

5. S. Schwarzinger, F. Brauer, P. Rösch, B. Schütz, B. Kämpf, G. Beckh, C. Lüllmann and A. Dübecke, "Authentic food: Why a single analysis parameter is not enough", Q\&More 1, 37-43 (2016).

6. P. C. Mahalanobis, "On the generalised distance in statistics", National Institute of Sciences of India 2, 49-55 (1936).
7. A. Surjushe, R. Vasani and D.G. Saple, "Aloe vera: A short review", Indian Journal of Dermatology 53, 163-6 (2008). doi: http://www.e-ijd. org/text.asp?2008/53/4/163/44785

8. X. Hu, Y. Shi, P. Zhang, M. Miao, T. Zhang and B. Jiang, "D-Mannose: Properties, Production, and Applications: An Overview", Comprehensive Reviews in Food Science and Food Safety 15(4), 669-826 (2016). doi: http://onlinelibrary.wiley.com/doi/10.1111/1541-4337.12211/full

9. T.M. Jyske, J.P. Suuronen, A.V Pranovich, T. Laakso, U. Watanabe, K. Kuroda and $\mathrm{H}$. Abe, "Seasonal variation in formation, structure, and chemical properties of phloem in Picea abies as studied by novel microtechniques", Planta Journal 242(3), 613-29 (2015). doi: http://link. springer.com/article/10.1007\%2Fs00425-015-2347-8

10. M. Spraul, B. Schütz, P. Rinke, S. Koswig, E. Humpfer, H. Schäfer, M. Mörtter, F. Fang, U.C. Marx and A. Minoja, "NMR-Based Multi Parametric Quality Control of Fruit Juices: SGF Profiling", Nutrients 1(2), 148-155 (2009). doi: http://www.mdpi.com/2072-6643/1/2/148

11. A. Maurizio and I. Grafl, "Das Trachtpflanzenbuch. Nektar und Pollen die wichtigsten Nahrungsquellen der Honigbiene“, Ehrenwith, Munich 2nd ed. 4, (1980). ISBN 3-431-02035-6

12. J. Liu, X.-P. Zhang and G. Duan, "Study on Mannose Formation during High Fructose Syrup Production", Journal of Food Science and Biotechnology 32(10), 1101-1105 (2013).

13. Hebi Higer Chemical Technology Co., LTD., "Ion exchange resins used for honey Mechanism of decolorization", (2004). doi: http://www. chemresin.com/en/NewsShow.asp?id=33

14. F. Helfferich, "Ion Exchange", McGraw-Hill, New York (1962). doi: http:// trove.nla.gov.au/work/3507138

15. D.W. Harris and M.S. Feather, "Studies on the Mechanism of the Interconversion of D-Glucose, D-Mannose, and D-Fructose in Acid Solution", Journal of American Chemical Society 97(1), 178-181 (1975). doi: http://pubs.acs.org/doi/abs/10.1021/ja00834a031?journalCode=jacsat

16. J.C. Jr. Speck, "The Lobry de Bruyn-Alberda van Ekenstein transformation", Advances in Carbohydrate Chemistry 13, 63-103 (1958). doi: http://dx.doi.org/10.1016/S0096-5332(08)60352-5 